\section{Covid-19 en pacientes renales crónicos: llamado de} atención

\section{Covid-19 in chronic kidney patients: a warning}

\section{Señor Editor:}

El brote de COVID-19 comenzó en noviembre de 2019 y el 11 de marzo de 2020 fue declarado una pandemia mundial por la Organización Mundial de la Salud. Los pacientes con COVID-19 tienen un mayor riesgo de mortalidad y falla multi-orgánica, y pueden experimentar síntomas debilitantes ${ }^{1,2}$. La pandemia ha impuesto una carga sin precedentes en los sistemas sanitarios de todo el mundo, con una demanda de cuidados críticos que exceden la capacidad de atención para este tipo de pacientes en varios países ${ }^{3,4}$. A la fecha, no existen vacunas que puedan prevenir la transmisión, ni tratamientos comprobados que sean efectivos para este tipo de infección viral ${ }^{5}$.

Los pacientes con enfermedades renales crónicas (en particular en diálisis o con trasplante renal), al contagiarse por COVID-19, tienen una mayor probabilidad de desarrollar complicaciones graves por múltiples razones. Muchos de ellos son de edad avanzada y tienen comorbilidades como enfermedades cardiovasculares, hipertensión arterial, diabetes, enfermedad pulmonar, además de tener un estado de compromiso inmunitario subyacente que se asocia a un mayor riesgo de morbimortalidad cuando se contagian con COVID-196 (ver Tabla). Los aspectos logísticos asociados a la terapia de hemodiálisis aumentan el riesgo de transmisión de la enfermedad, debido a la necesidad de los pacientes de concurrir trisemanalmente a centros de atención médica, donde la proximidad física de los pacientes durante sus sesiones de hemodiálisis es difícil de evitar. Resulta fundamental implementar diversas estrategias preventivas en las instalaciones de hemodiálisis, con el fin de disminuir precozmente los riesgos de contagio de los pacientes en este tipo de terapias ${ }^{6}$.

Frente al enorme desafío que significa enfrentar esta pandemia en nuestro país, la Sociedad Chilena de Nefrología, en conjunto con la Fundación Pro Salud Renal, la Asociación de Pacientes Dializados y Trasplantados de Chile, y la Sociedad de Enfermería en Diálisis y Trasplante, crearon el 29 de marzo 2020 una Fuerza de Tarea Conjunta Renal Anti COVID-19 (FUTAC-RENAL), con el objetivo de desarrollar y potenciar todas aquellas medidas basadas en la mejor evidencia científica disponible, así como aquellas de mejor costo-efectividad comprobadas con la finalidad de evitar la propagación y contagio del COVID-19 en pacientes con patología renal y en el personal de salud que los atiende ${ }^{8}$. A la fecha, la FUTAC-Renal ha estado apoyando de manera concreta a los distintos equipos clínicos que trabajan con pacientes renales crónicos a lo largo de todo el país, a través de: capacitaciones y seminarios online; generación de información epidemiológica fidedigna (ver gráficos al 27/4/2020); recomendaciones basadas en la evidencia disponible para el manejo de COVID-19 en pacientes en diálisis y trasplantados; y potenciado todas las medidas de cuidado y protección del personal de salud a cargo de estos pacientes (ver www.nefro.cl/ covid-19). Complementando las acciones anteriores, la Fundación Pro Salud Renal presentó un proyecto a la Fundación VientoSur para ir en ayuda directa de pacientes y del personal clínico de las 262 unidades de hemodiálisis de Chile, haciendo entrega de Elementos de Protección Personal (EPP). Esta solicitud fue aprobada a fines de marzo, iniciando el envío de esta ayuda a las unidades de diálisis desde mediados de abril.

Tenemos la convicción que, en tiempos difíciles 


\section{COMPARACIÓN CON POBLACIÓN GENERAL}

\begin{tabular}{|c|c|c|}
\hline & 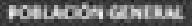 & 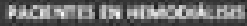 \\
\hline foem acanosa & 1045128 & 2ising \\
\hline 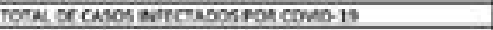 & 1405 & ri \\
\hline 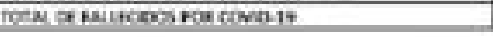 & 398 & 3 \\
\hline 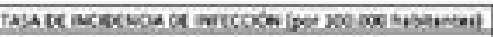 & 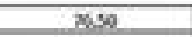 & 31501 \\
\hline 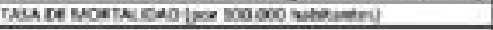 & 111 & 21200 \\
\hline 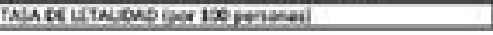 & $14 \mathrm{~s}$ & sen \\
\hline \multicolumn{3}{|c|}{ 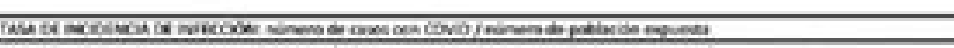 } \\
\hline \multicolumn{3}{|c|}{ 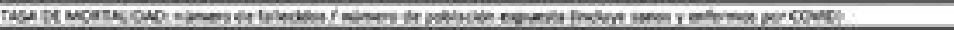 } \\
\hline \multicolumn{3}{|c|}{ 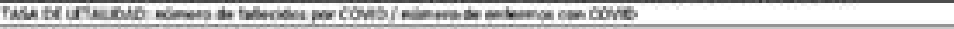 } \\
\hline \multicolumn{3}{|c|}{ 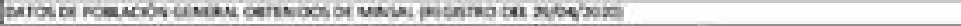 } \\
\hline
\end{tabular}

PACIENTES EN HEMODIÁLISIS VERSUS POBLACIÓN GENERAL

Aumento de tasa de infección: $4,1 \mathrm{x}$

Aumento de tasa de mortallidad: $18,9 \mathrm{x}$

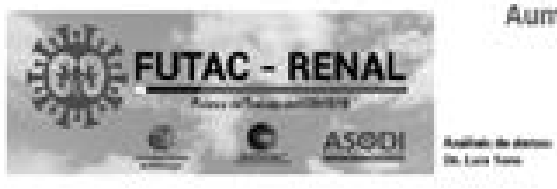

Aumento de tasa de letalidad: $4,6 x$

como éste, iniciativas como las descritas son del todo necesarias y debiesen ser consideradas un refuerzo a las medidas dictadas por las autoridades sanitarias del país. Al sumar esfuerzos de Organizaciones de Pacientes, Sociedades Científicas, y Fundaciones, hemos podido informar, capacitar, entregar recomendaciones, y canalizar recursos económicos para ir en ayuda directa a pacientes y personal clínico, en un momento crucial y crítico para todos.

\section{Rubén Torres Díaz ${ }^{1}$ Eduardo Lorca Herrera ${ }^{2}$ ${ }^{1}$ Presidente, Sociedad Chilena de Nefrología. ${ }^{2}$ Presidente, Fundación Pro Salud Renal.}

\section{Referencias}

1. Zhou F, Yu T, Du R, Fan G, Liu Y, Liu Z, et al. Clinical course and risk factors for mortality of adult inpatients with COVID-19 in Wuhan, China: a retrospective cohort study. Lancet 2020; 395(10229): 1054-62.

2. Chen N, Zhou M, Dong X, Qu J, Gong F, Han Y, et al. Epidemiological and clinical characteristics of 99 cases of 2019 novel coronavirus pneumonia in Wuhan, China: a descriptive study. Lancet 2020; 395 (10223): 507-13.

3. Lee CCM, Thampi S, Lewin B, Lim TJD, Rippin B, Wong WH, Agarwal RV. Battling COVID-19: Critical care and pe- ri-operative healthcare resource management strategies in a tertiary academic medical centre in Singapore. Anaesthesia 2020: Apr 8. doi: 10.1111/anae.15074.

4. García-Castrillo L, Petrino R, Leach R, Dodt C, Behringer W, Khoury A, et al. European Society For Emergency Medicine position paper on emergency medical systems response to COVID-19. Eur J Emerg Med 2020: Apr 1. doi: 10.1097/ MEJ.0000000000000701.

5. Sanders JM, Monogue ML, Jodlowski TZ, Cutrell JB. Pharmacologic treatments for coronavirus disease 2019 (COVID-19): a review. JAMA 2020; Apr 13. doi: 0.1001/ jama.2020.6019.

6. Alp Ikizler T. COVID-19 and Dialysis Units: What Do We Know Now and What Should We Do?. American Journal of Kidney Diseases. 2020 March 17. doi.org/10.1053/j. ajkd.2020.03.008.

Correspondencia a:

Dr. Eduardo Lorca $\mathrm{H}$.

Departamento de Medicina Interna Oriente

Facultad de Medicina de la Universidad de Chile

Avenida Salvador 486, Providencia. Santiago. Chile.

elorca@med.uchile.cl 